\title{
Ga interstitial stability and its effect on the electronic properties of $\beta-\left(\mathrm{Al}_{\mathbf{x}} \mathrm{Ga1}-\mathrm{x}\right)_{2} \mathrm{O} 3$ alloy
}

\author{
Adrian Chmielewski ${ }^{1}$, Ziling Deng ${ }^{2}$, Yuewei Zhang ${ }^{3}$, Akhil Mauze ${ }^{3}$, Wolfgang Windl ${ }^{2}$ and Nasim \\ Alem $^{4}$
}

${ }^{1}$ Penn State University, State College, Pennsylvania, United States, ${ }^{2}$ Ohio State University, United States, ${ }^{3}$ University of California Santa Barbara, United States, ${ }^{4}$ Pennsylvania State University, United States

Alloying $\beta-G a 2 \mathrm{O} 3$ with $\mathrm{A} 12 \mathrm{O} 3$ to form a $\beta$-(AlxGa1-x)2O3 structure permits to modulate the bandgap consequently offering the ability to control the electronic properties of devices based on this material [1]. Although a huge progress has been made by the growers' community in the fabrication of a stable $\beta$ (AlxGa1-x)2O3 alloy using various growing techniques [2], the capability to grow this material with well controlled defects remain a challenge. In addition, increasing the amount of $\mathrm{Al}$ often results in the formation of even more defects in the $\beta$-(AlxGa1-x)2O3 crystal structure [3] and one of them is Ga interstitial (Gai) that sits in between two Ga atoms on the tetrahedral site. Although many Gai observations, little is known about the structural distortions and the stability of the defect and its resulting electronic properties in the $\beta$-(AlxGa1-x)2O3 crystal. Moreover, addition of $\mathrm{Al}$ can modulate the electronic structure of the crystal by tuning the band gap and introducing changes in the band structure.

This work presents a systematic study of the stability of Gai depending of its nearest atomic environments. This study uses high angle annular dark field scanning transmission electron microscopy (HAADF-STEM) imaging to probe the Ga interstitials and uncover the chemical and atomic structure of these interstitial defects. Using density functional theory (DFT) calculations, several different $\beta$-(AlxGa1$\mathrm{x}) 2 \mathrm{O} 3$ structures containing Gai surrounded by a few $\mathrm{Al}$ atoms are generated and compared with experimental findings. Figure 1.a shows a simulated structure of $\beta$-(Al0.2Ga0.8)2O3 supercell in which a Gai is surrounded by two Al atoms. Figure 1.b shows an experimental HAADF-STEM image of a Gai atom potentially representing the same atomic structure as predicted by DFT calculations. Our investigations show that the presence of two $\mathrm{Al}$ atoms affects the position of the Ga interstitial atom and its nearest Ga neighbor. Moreover, the effect of the Gai stability on the electronic properties of $\beta$-(AlxGa1$\mathrm{x}) 2 \mathrm{O} 3$ will also be discussed. Using electron energy loss spectroscopy (EELS), we have probed the electronic structure of the sample and the variations in the band gap that is introduced through addition of Al. According to density of states calculations the deep level states created by Gai (shown by the blue arrow in Figure 1.c) is directly shifted by its surrounding number of $\mathrm{Al}$ atoms. These findings provide great insight into the fabrication of defects-controlled $\mathrm{AlGaO}$ based devices.

Acknowledgements:

The work on the wide bang gap systems at PSU was supported by the Air Force Office of Scientific Research (AFOSR) program FA9550-18-1-0277 as well as GAME MURI, 10059059-PENN. 

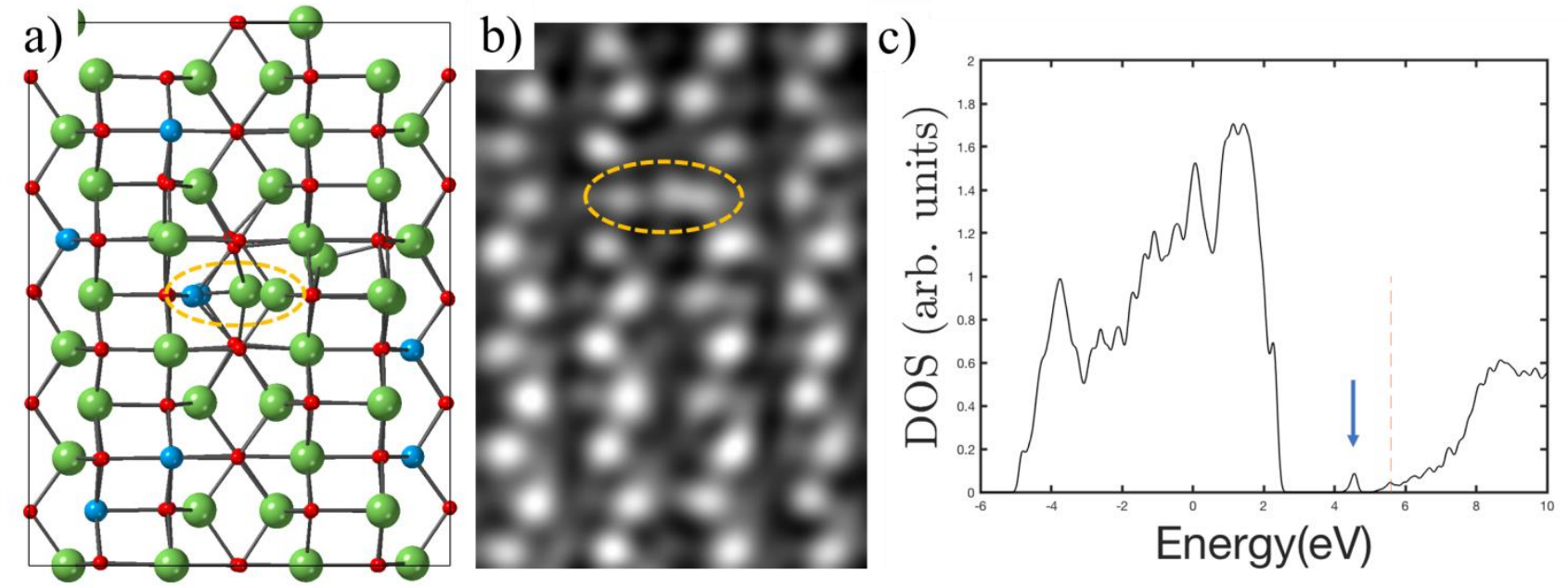

Figure 1. (a) Crystal structure model of Gai in (Al0.2Ga0.8)2O3 with two $\mathrm{Al}$ atoms surrounding the Gai. (b) Its corresponding high resolution HAADF-STEM image. The Gai is highlighted by the orange dashed ellipse. (c) Density of states calculations showing a deep level state (around $\sim 4.5 \mathrm{eV}$, indicated by the blue arrow) that is attributed to the presence of the Gai.

\section{References}

[1] W. Hu et al., Journal of Alloys and Compounds 864, 158765 (2021)

[2] S.J Pearton et al., Applied Physics Reviews 5, 011301 (2018)

[3] B. Fu et al., J. Semicond. 40, 011804 (2019) 